\title{
Local feedback passivation of nonlinear discrete-time systems through the speed-gradient algorithm 岤, 论抎
}

\author{
Eva M. Navarro-López* \\ Escuela Técnica Superior de Ingenieros Industriales, Universidad de Castilla-La Mancha, Avda. Camilo José Cela s/n, 13071 Ciudad Real, Spain
}

Received 1 August 2005; received in revised form 28 August 2006; accepted 9 December 2006

Available online 9 May 2007

\begin{abstract}
This paper proposes a method in order to render passive discrete-time nonlinear multiple-input multiple-output (MIMO) systems which are affine in control. The methodology is based on the discrete-time version of the speed-gradient (SG) algorithm. For the application of the SG algorithm, quasi- $V$-passive and feedback quasi- $V$-passive systems are introduced. Two kinds of feedback laws rendering the system locally quasi- $V$-passive are obtained: a dynamic one and a static one. The feedback passivation methodology is applied to two examples. Some frequency-domain properties of the feedback transformed system are highlighted for the linear example.
\end{abstract}

(C) 2007 Elsevier Ltd. All rights reserved.

Keywords: Discrete-time systems; Nonlinear systems; Energy control; Passive elements; Feedback stabilization

\section{Introduction}

Passive systems are endowed with highly desirable stability properties which may simplify systems analysis and control design (Hill \& Moylan, 1980). This fact impels to transform a system which is not passive into a passive one. The action of rendering a system passive by means of a state feedback is known as feedback passivation. Systems which can be rendered passive are referred to as feedback passive systems.

Passivity and dissipativity implications in continuous-time systems have been broadly studied. Nevertheless, many problems concerning dissipativity and passivity in the nonlinear discrete-time setting remain unsolved, or they have not attracted as significant attention as in the continuous-time case. This is the case of the feedback passivation problem.

Several results concerning feedback passivation and feedback dissipative systems in the nonlinear discrete-time setting

\footnotetext{
it This is a modified version of that presented at the 16th IFAC World Congress, Prague, 2005. This work has been partially supported by the Ministerio de Educación y Ciencia (MEC) of Spain under Ramón y Cajal research contract.

访败 This paper was recommended for publication in revised form by Associate Editor Dragan Nesic under the direction of Editor André Tits.

* Tel.: +34667927042.

E-mail address: navarro.lopez@gmail.com.
}

have been reported in the literature. Necessary and sufficient conditions are proposed in Byrnes and Lin (1994) and Navarro-López (2007) for the feedback losslessness and feedback passivation cases, respectively, for multiple-input multiple-output (MIMO) affine-in-input nonlinear discretetime systems with the restriction of considering storage functions $V$ such that $V(f(x)+g(x) u)$ is quadratic in $u$. These works are based on the properties of the relative degree and zero dynamics of the nonpassive system and the approach is inherited from the continuous-time case (Byrnes, Isidori, \& Willems, 1991). These results were extended in Navarro-López and Fossas-Colet (2004) to general systems without requiring $V(f(x, u))$ to be quadratic in $u$. The feedback dissipativity property is treated for single-input single-output (SISO) nonlinear discrete-time systems of general form in Navarro-López, Sira-Ramírez, and Fossas-Colet (2002).

In the present paper, the feedback passivation problem is considered for MIMO nonlinear discrete-time systems which are affine in the input. A class of passive systems referred to as quasi- $V$-passive systems is used. An alternative method to those appeared in the literature for aforementioned systems to be rendered locally feedback quasi- $V$-passive is proposed and the $\mathrm{SG}$ algorithm in its discrete-time version is used. Two kinds of solutions are obtained. The problem of feedback passivation 
for continuous-time nonlinear systems is solved by means of the speed-gradient (SG) algorithm, for example, in Fradkov (1991). The discrete-time version of the SG methodology is given in Fradkov and Pogromsky (1998).

The results presented could be adapted for stabilization purposes together with techniques, such as, the ones presented in Jiang, Lin, and Wang (2004) or the Energy Shaping and Damping Injection, which was proposed for the discrete-time general case in Navarro-López et al. (2002).

The paper is focused on nonlinear systems which are affine in the control. Models of this type describe a great variety of systems. Most of them represent approximate discretizations of continuous-time systems. See, for example, Navarro-López (2007) where a class of bilinear systems describing different approximate discretized systems is presented. In the last few years, there has been an increasing interest in discrete approximate representations of continuous-time systems. For instance, the control of continuous-time processes by means of discretetime approximate models of the plant (Nešić \& Grüne, 2005; Nešić \& Teel, 2004), or the use of new hybrid representations to describe complex dynamical systems (van der Schaft \& Schumacher, 2000). Most of these examples may be described by the class of systems studied here.

\section{Basic definitions}

Let a system of the form

$$
\begin{aligned}
& x(k+1)=f(x(k))+g(x(k)) u(k), \\
& y(k)=h(x(k))+J(x(k)) u(k),
\end{aligned}
$$

where $f(x), g(x), h(x), J(x)$ are smooth maps and $f(x) \in$ $\mathscr{X} \subset \mathbb{R}^{n}, g(x) \in \mathscr{G} \subset \mathbb{R}^{n \times m}, h(x) \in \mathscr{Y} \subset \mathbb{R}^{m}, J(x) \in \mathscr{J} \subset$ $\mathbb{R}^{m \times m}, x \in \mathscr{X}, u \in \mathscr{U} \subset \mathbb{R}^{m}, k \in \mathbb{Z}_{+}=\{0,1,2, \ldots\}$. All considerations are restricted to an open set of $\mathscr{X} \times \mathscr{U}$ containing $(\bar{x}, \bar{u})$, having $\bar{x}$ as an isolated fixed point of $f(x)+g(x) \bar{u}$ with $\bar{u}$ constant, i.e., $f(\bar{x})+g(\bar{x}) \bar{u}=\bar{x}$.

A positive definite $\mathscr{C}^{2}$ function $V: \mathscr{X} \rightarrow \mathbb{R}$ such that $V(0)=$ $0 \Longleftrightarrow x=0$ is addressed as storage function. Another $\mathscr{C}^{2}$ function $\phi: \mathscr{X} \times \mathscr{U} \rightarrow \mathbb{R}$, such that $\phi(\cdot, u)$ is positive for each $u \in \mathscr{U}$, with $\phi(0,0)=0$, is referred to as dissipation rate function in the sense proposed in Hill and Moylan (1980) and Navarro-López et al. (2002).

The application of the SG method gives rise to two types of feedback laws. Let $\alpha: \mathscr{X} \times \mathscr{U} \rightarrow \mathscr{U}$ be a smooth function. A static state feedback law $u=\alpha(x, v)$ is regular if $\forall(x, v) \in$ $\mathscr{X} \times \mathscr{U}$ it follows that $\partial \alpha / \partial v$ is invertible. Let $\beta: \mathscr{X} \times \mathscr{U} \times \mathscr{U} \rightarrow$ $\mathscr{U}$ be a smooth function. A dynamic feedback law $u(k+1)=$ $\beta(x(k), u(k), v(k))$ is regular if $\partial \beta / \partial v$ is invertible $\forall(x, u, v) \in$ $\mathscr{X} \times \mathscr{U} \times \mathscr{U}$. System (1) with $u(k)=\alpha(x(k), v(k))$ or $u(k+1)=$ $\beta(x(k), u(k), v(k))$ is referred to as the feedback transformed system.

In order to use the SG algorithm for feedback passivation purposes, the following definitions are introduced, which are based on the definitions given in Byrnes and Lin (1994).
Definition 1. System (1) with storage function $V(x)$ is said to be locally quasi- $V$-passive if there exist a dissipation rate function $\phi$ and a constant $\Delta>0$ such that

$$
\begin{aligned}
& V(f(x)+g(x) u)-V(x)-[h(x)+J(x) u]^{\mathrm{T}} u \\
& \quad+\phi(x, u) \leqslant \Delta, \quad \forall(x, u) \in \mathscr{X} \times \mathscr{U} .
\end{aligned}
$$

Definition 2. Consider system (1). Assume that there exists a storage function $V(x)$. The system is said to be locally feedback quasi- $V$-passive if there exists a regular static state feedback law $u(k)=\alpha(x(k), v(k))$ or a regular dynamic feedback law $u(k+1)=\beta(x(k), u(k), v(k))$, with $v$ as the new input, such that the feedback transformed system is locally quasi- $V$-passive.

\section{The SG algorithm and the local feedback passivation}

The discrete-time version of the SG method (Fradkov \& Pogromsky, 1998) is formulated as finding a control law $u(k)$ which ensures the control objective,

$Q(x(k+1)) \leqslant \Delta \quad$ when $k>k^{*}$,

with some nonnegative $\mathscr{C}^{1}$ control goal function $Q$, a threshold value $\Delta>0$ and some $k^{*} \in \mathbb{Z}_{+}$.

In this section, the discrete-time version of the SG algorithm is adapted to propose a method in order to render systems of the form (1) locally feedback quasi- $V$-passive. For this purpose, the following control goal function is considered:

$$
\begin{aligned}
Q_{d}(x(k), u(k), v(k))= & V(x(k+1))-V(x(k))-y^{\mathrm{T}}(k) v(k) \\
& +\phi(x(k), u(k)), \quad v \in \mathscr{U} .
\end{aligned}
$$

The methodology and its applicability conditions are based on the results given in Fradkov and Pogromsky (1998). Let us define

$Q_{d, k}(u):=Q_{d}(x(k), u(k), v(k))$.

Assume that $v(k)$ is constant $\forall k$. The partial derivative of $Q_{d}$ with respect to $u$ is denoted by $\nabla_{u} Q_{d, k}(u)$.

Proposition 3. Let a system of the form (1). Consider $V(x)$ as the storage function and a $\mathscr{C}^{1}$ control goal function $Q_{d}$ as defined in (4) with the dissipation rate function $\phi$ such that makes $Q_{d}$ be nonnegative, $\forall u, v \in \mathscr{U}$. Let $\Delta$ and $\varepsilon^{*}$ be positive constants such that $\Delta$ and $\varepsilon^{*}$ are close to zero with $\varepsilon^{*} \ll \Delta$, $1-\varepsilon^{*} / \Delta \approx 1$, and $u_{0}=u(0), u_{*} \in \mathscr{U}$ are constant vectors. Assume that:

A1. There exist $\varepsilon^{*}>0$ and a vector $u^{*}$ such that

$$
Q_{d, k}\left(u^{*}\right) \leqslant \varepsilon^{*}<\Delta .
$$

A2. For all $u \in \mathscr{U}$ :

$$
\left(u^{*}-u\right)^{\mathrm{T}} \nabla_{u} Q_{d, k}(u) \leqslant Q_{d, k}\left(u^{*}\right)-Q_{d, k}(u)<0 .
$$

A3. For any $\rho>0$ and any $k \in \mathbb{Z}_{+}$, there exists $\kappa(\rho)>0$ such that the following inequality is satisfied:

$\left|\nabla_{u} Q_{d, k}(u)\right|^{2} \leqslant \kappa(\rho)$,

as long as $\left|u-u^{*}\right| \leqslant \rho$ and $Q_{d, k}(u)>\Delta$. 
Then, for any $u_{0}$, there exists a $k^{*} \in \mathbb{Z}_{+}$such that the system is locally feedback quasi-V-passive for $k>k^{*}$ by means of a dynamic feedback of the form

$u(k+1)=u(k)-\gamma(k) \nabla_{u} Q_{d, k}(u)$,

$\gamma(k)=\gamma_{c} \delta(k)\left|\nabla_{u} Q_{d, k}(u)\right|^{-2}, \quad 0<\gamma_{c}<2$,

$\delta(k)= \begin{cases}Q_{d, k}(u) & \text { if } Q_{d, k}(u) \geqslant \Delta, \\ 0 & \text { otherwise. }\end{cases}$

Proof. The proof follows the same arguments as the ones given for the SG discrete-time version (Fradkov \& Pogromsky, 1998). Let us define $V_{d}(u(k)):=\left|u(k)-u^{*}\right|^{2}$ and $\Delta V_{d}=V_{d}(u(k+$ $1))-V_{d}(u(k))$. Suppose that $Q_{d, k}(u) \geqslant \Delta$. Then:

$\Delta V_{d}=(u(k+1)-u(k))^{\mathrm{T}}\left[2\left(u(k)-u^{*}\right)+(u(k+1)-u(k))\right]$.

Considering (9a), $\Delta V_{d}$ yields to

$\Delta V_{d}=2 \gamma(k)\left(u^{*}-u(k)\right)^{\mathrm{T}} \nabla_{u} Q_{d, k}(u)+\gamma^{2}(k)\left|\nabla_{u} Q_{d, k}(u)\right|^{2}$.

Using (7) and substituting $\gamma(k)$ in the above expression, it is obtained:

$$
\begin{aligned}
\Delta V_{d} & \leqslant-\frac{\gamma_{c} Q_{d, k}^{2}(u)}{\left|\nabla_{u} Q_{d, k}(u)\right|^{2}}\left\{2 \frac{Q_{d, k}(u)-Q_{d, k}\left(u^{*}\right)}{Q_{d, k}(u)}-\gamma_{c}\right\} \\
& \leqslant-\frac{\gamma_{c} \Delta}{\left|\nabla_{u} Q_{d, k}(u)\right|^{2}}\left[2\left(1-\frac{\varepsilon^{*}}{\Delta}\right)-\gamma_{c}\right] \leqslant 0 .
\end{aligned}
$$

By assumption $\mathrm{A} 3,\left|\nabla_{u} Q_{d, k}(u)\right|^{2}$ is bounded. Then, $V_{d}$ is bounded $\forall k$, and consequently, so is $u(k), \forall k$. Indeed, $u(k) \rightarrow$ $u^{*}$ as $k$ increases. In addition, the relation $\delta(k) \neq 0$ can be satisfied for no more than a finite number of $k \leqslant k^{*}$, after that $\delta(k)=0, u(k)=$ constant,$\forall k>k^{*}$ and $Q_{d, k}(u)<\Delta$. Then, inequality (2) is satisfied and the feedback transformed system is locally quasi- $V$-passive.

As it is established in Fradkov and Pogromsky (1998), the SG-based control scheme can be also given for a static feedback.

Proposition 4. Consider $V(x)$ as the storage function and $a \mathscr{C}^{1}$ control goal function $Q_{d}$ as defined in (4) with the dissipation rate function $\phi$ such that makes $Q_{d}$ be convex and nonnegative, $\forall u, v \in \mathscr{U}$. Suppose that the magnitude of vector $\nabla_{u} Q_{d, k}(u)$ is nonincreasing along solutions of system (1), and assumptions A1, A3 of Proposition 3 are met. Let $\Delta$ and $\varepsilon^{*}$ be positive constants, and $u_{*} \in \mathscr{U}$ is a constant vector. Then, there exists a $k^{*} \in \mathbb{Z}_{+}$such that system (1) is locally feedback quasi$V$-passive for $k>k^{*}$ by means of a regular feedback $u(k)=$ $\alpha(x(k), v(k))$, obtained from the relation:

$u(k)=-\gamma_{c s} \nabla_{u} Q_{d, k}(u)+u^{*}$,

with $\gamma_{c s}$ a positive constant ensuring the local asymptotic stability of the fixed point of the feedback transformed system when $v=0$, and $u^{*}$ a constant vector as defined in (6).
Proof. Let us consider $V_{d}(u(k))$ and $\Delta V_{d}$ as defined above. Using (10), one yields to

$\Delta V_{d}=\gamma_{c s}^{2}\left[\left|\nabla_{u} Q_{d, k+1}(u)\right|^{2}-\left|\nabla_{u} Q_{d, k}(u)\right|^{2}\right]$.

Since $\left|\nabla_{u} Q_{d, k}(u)\right|$ is a nonincreasing function along solutions of system (1), then $\Delta V_{d} \leqslant 0$. Consequently, following the same arguments as in the proof of Proposition 3, there exists $k^{*} \in \mathbb{Z}_{+}$ for which the feedback transformed system is locally quasi- $V$ passive $\forall k>k^{*}$.

The feedback passivation methodology, in its two versions, will be applied to render two quasi- $V$-passive examples in the next two sections. Although the feedback passivation methodology is applicable to MIMO systems, the examples are SISO for simplicity's sake in computations.

\section{The linear discretized buck converter model}

Let us consider a DC-to-DC buck converter. This circuit contains a switch which can be in two positions (1 or 0 ). A discretized model of a normalized averaged DC-to-DC buck converter is (Navarro-López et al., 2002)

$x(k+1)=A x(k)+B u(k)$,

$y(k)=C x(k)+D u(k)$,

with,

$A=\left(\begin{array}{cc}a & -b \\ b & c\end{array}\right), \quad B=\left(\begin{array}{c}(1-a) \gamma_{b}+b \\ 1-b \gamma_{b}-c\end{array}\right), \quad C=(0,1), D=1$,

with $u=t_{\text {on }} / T \in[0,1], t_{\text {on }}$ the time the switch is in 1 position, and $T$ the commutation period. $x=\left(x_{1}, x_{2}\right)^{\mathrm{T}}$, $x_{1}$ is a normalized current, $x_{2}$ is a normalized voltage, $\gamma_{b}=\sqrt{L_{b}} / R_{b} \sqrt{C_{b}}=0.353553$ is the normalized load. Constants $a=0.9406416964, b=0.3254699438, c=0.8255706942$ are obtained with: $V_{\text {in }}=407 \mathrm{~V}$ (power supply voltage), $L_{b}=1 \mathrm{mH}$, $C_{b}=80 \mu \mathrm{F}, R_{b}=10 \Omega, f_{c}=10 \mathrm{kHz}$ (switch commutation frequency), $T=0.3535533906 \mathrm{~s}$.

The system is not passive and the goal is to render it locally quasi- $V$-passive having as storage function $V=(\eta / 2)\left(x_{1}^{2}+x_{2}^{2}\right)$, with $\eta=C_{b} V_{\text {in }}^{2}=13.25192$. Function $\phi(x, u)$ is chosen as

$$
\begin{aligned}
\phi(x, u)= & \eta \mu\left\{x_{1}^{2}\left(a^{2}+b^{2}\right)+x_{2}^{2}\left(b^{2}+c^{2}\right)+u^{2}\left[\gamma_{b}^{2}(1-a)^{2}\right.\right. \\
& \left.\left.+b^{2}\left(1+\gamma_{b}^{2}\right)+(1-c)^{2}\right]\right\},
\end{aligned}
$$

with $\mu$ a positive constant. Let $x_{1} \in\left[0, \gamma_{b} \rho\right], x_{2} \in[0, \rho], u, v \in$ $[0,1]$, and $\rho>1$. For the example:

$Q_{d}\left(x_{1}, x_{2}, u, v\right)=a_{u} u^{2}+b_{u}\left(x_{1}, x_{2}, v\right) u+c_{u}\left(x_{1}, x_{2}, v\right)$,

where $a_{u}$ and $b_{u}, c_{u}$ are a constant and two functions, respectively, depending on $\eta, \mu$ and system parameters. $b_{u}, c_{u}$ are linear in the states and control $v$.

It is necessary to assure that the function $Q_{d}$ is positive, which will be achieved by means of choosing $\mu$. In addition, conditions of Propositions 3 and 4 must be checked. 
First, a way of assuring the positiveness of $Q_{d}$ is by assuring that $Q_{d}$ is a positive function for all the candidates for local minima, and this is achieved for $\mu>\bar{\mu}=0.296853$. Second, from the fact that for $\mu>\bar{\mu}$, it follows that $Q_{d}>0$ and $Q_{d}$ has a relative minimum at $x=0, u=0$ with $v=\bar{v}$ a constant. The condition in $\mathrm{A} 1$ is met due to the fact that $Q_{d}$ is positive with a relative minimum at $(x=0, u=0, \bar{v})$, then a constant $0<\varepsilon^{*}<\Delta$ can be always found and a control $u^{*}$ for which $Q_{d}$ is smaller. Thus, $Q_{d, k}(u) \leqslant \Delta, \forall k>k^{*}$. This $u^{*}$ can be $u^{*}=0$ considering $x_{1}, x_{2}, v$ fixed near to zero. On the other hand, condition (7) is met considering $u^{*}=0, v$ small enough and $\mu$ high enough. Indeed, condition (7) is met due to the convexity of $Q_{d}$ with $\mu>\bar{\mu}$. Condition (8) is met considering the property of uniform continuity in a set (Marsden \& Hoffman, 1993). Then, two feedback passivation schemes are obtained:

(1) From (9), a dynamic feedback law:

$$
\begin{aligned}
& u(k+1)=u(k)-\gamma(k)\left[2 a_{u} u(k)+b_{u}\left(x_{1}(k), x_{2}(k), v(k)\right)\right], \\
& \gamma(k)=\gamma_{c} \delta(k)\left|2 a_{u} u(k)+b_{u}\left(x_{1}(k), x_{2}(k), v(k)\right)\right|^{-2}, \\
& 0<\gamma_{c}<2, \quad \delta(k)= \begin{cases}Q_{d, k}(u) & \text { if } Q_{d, k}(u) \geqslant \Delta, \\
0 & \text { otherwise. }\end{cases}
\end{aligned}
$$

(2) From (10), a static feedback law:

$$
u(k)=-\frac{\gamma_{c s} b_{u}\left(x_{1}(k), x_{2}(k), v(k)\right)}{1+2 \gamma_{c s} a_{u}},
$$

with $\gamma_{c s}>0$ ensuring the fixed point of the transformed system to be asymptotically stable when $v=0$.

Passive systems exhibit special features in the frequency domain. Passivity, for linear systems, is equivalent to positive realness of a transfer function. The same is for the discrete-time case (Hitz \& Anderson, 1969). Quasi- $V$-passive systems also exhibit special frequency-domain characteristics, which can be identified in the example.

The Nyquist diagrams of the pulse transfer functions for system (11) and for the feedback transformed system obtained with $\mu=0.3$ and $\gamma_{c s}=0.7$ are depicted in Fig. 1. The Nyquist plot of the quasi- $V$-passive system lies on the right-hand side half plane, but it does not touch the axis $\operatorname{Re}\left[G\left(\mathrm{e}^{\mathrm{j} \omega}\right)\right]=0$, it is located to the right of $\operatorname{Re}\left[G\left(\mathrm{e}^{\mathrm{j} \omega}\right)\right]=\Delta$.

\section{A nonlinear example}

Consider a system of the form (Sira-Ramírez, 1991)

$$
\begin{aligned}
& x_{1}(k+1)=\left[x_{1}^{2}(k)+x_{2}^{2}(k)+u(k)\right] \cos \left[x_{2}(k)\right], \\
& x_{2}(k+1)=\left[x_{1}^{2}(k)+x_{2}^{2}(k)+u(k)\right] \sin \left[x_{2}(k)\right], \\
& y(k)=x_{1}^{2}(k)+x_{2}^{2}(k)+u(k) .
\end{aligned}
$$

The goal is to render system (16) locally quasi- $V$-passive with a storage function $V=x_{1}^{2}+x_{2}^{2}$. Function $\phi(x, u)$ is chosen in order to collect the positive terms of $V(x(k+1))$ :

$\phi(x, u)=\mu\left[\left(x_{1}^{2}+x_{2}^{2}\right)^{2}+u^{2}+x_{1}^{2}+x_{2}^{2}\right]$, (i)

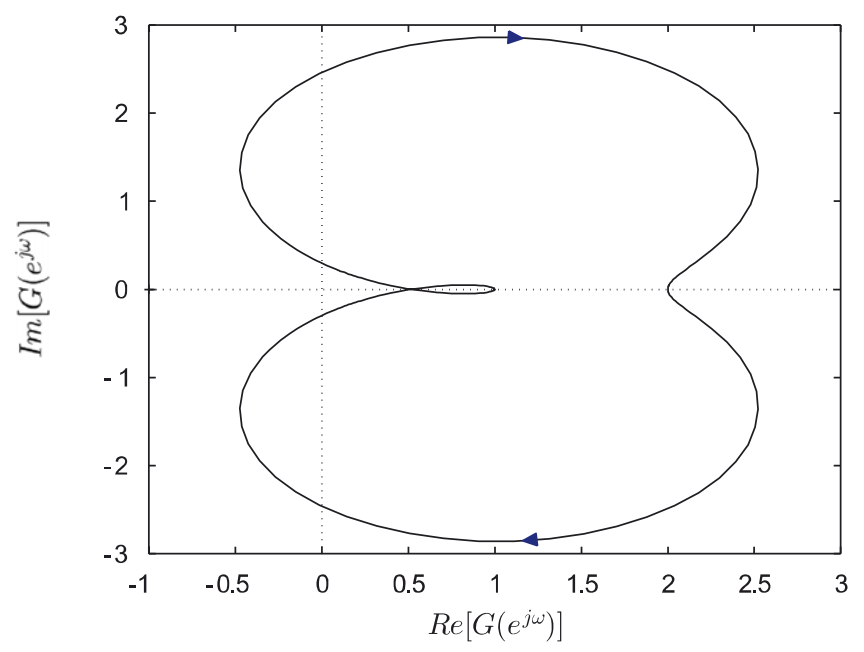

$($ ii)

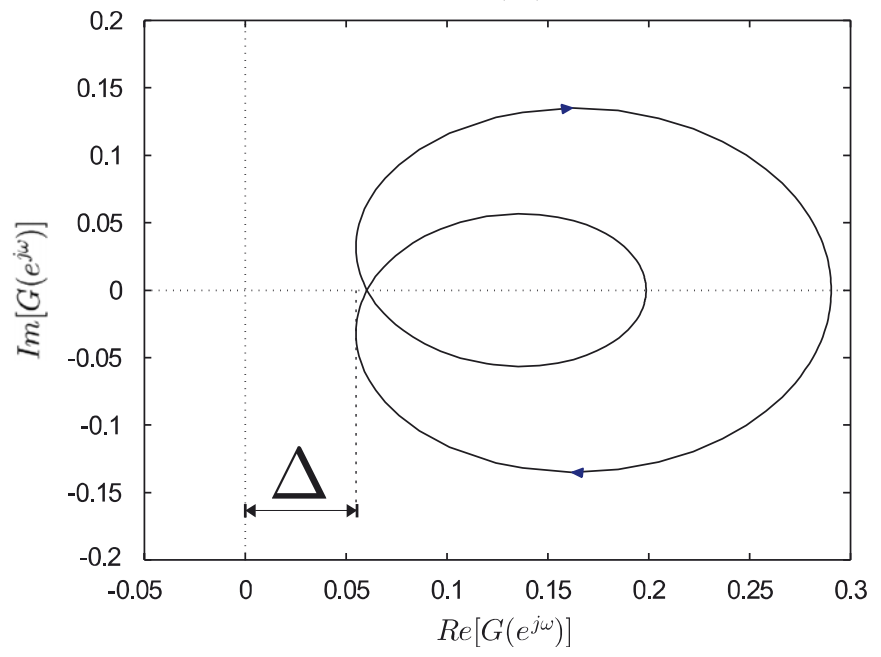

Fig. 1. Buck-converter Nyquist diagrams: (i) for the exact discretized model with $y=x_{2}+u$; (ii) for the quasi- $V$-passive system.

with $\mu>0$. Suppose $x_{1}, x_{2} \in\left[-\rho_{x}, \rho_{x}\right], u \in\left[-\rho_{u}, \rho_{u}\right], v \in$ $\left[-\rho_{v}, \rho_{v}\right]$, with $\rho_{x}, \rho_{u}, \rho_{v}$ positive constants. Thus,

$$
\begin{aligned}
Q_{d}\left(x_{1}, x_{2}, u, v\right)= & u^{2}(1+\mu)+2 u\left(x_{1}^{2}+x_{2}^{2}\right)+\left(x_{1}^{2}+x_{2}^{2}\right) \\
& \times\left[(1+\mu)\left(x_{1}^{2}+x_{2}^{2}\right)+\mu-1\right]-y v .
\end{aligned}
$$

Function $Q_{d}$ is assured to be positive in all candidates for local minima in the domain of definition of $Q_{d}$ with $\mu>1$. In addition, with this $\mu$, the critical point $\left(x_{1}, x_{2}, u, v\right)=(0,0,0, \bar{v})$, with $\bar{v}$ a constant, is assured to be a relative minimum of $Q_{d}$.

Following the same procedure as the one given for the linear example, when $\mu>1$, the conditions of Propositions 3 and 4 are verified. Two passifying controls are then obtained:

(1) A dynamic feedback:

$$
\begin{aligned}
u(k+1)= & u(k)-\gamma(k)\{2 u(k)(1+\mu) \\
& \left.+2\left[x_{1}^{2}(k)+x_{2}^{2}(k)\right]-v(k)\right\},
\end{aligned}
$$




$$
\begin{aligned}
\gamma(k)= & \gamma_{c} \delta(k) \mid 2 u(k)(1+\mu) \\
& +2\left[x_{1}^{2}(k)+x_{2}^{2}(k)\right]-\left.v(k)\right|^{-2}, \\
\delta(k)= & \begin{cases}Q_{d, k}(u) & \text { if } Q_{d, k}(u) \geqslant \Delta, \\
0 & \text { otherwise, }\end{cases}
\end{aligned}
$$

with $\mu>1$ and $0<\gamma_{c}<2$.

(2) A static feedback:

$$
u(k)=-\frac{\gamma_{c s}\left[2 x_{1}^{2}(k)+2 x_{2}^{2}(k)-v(k)\right]}{1+2 \gamma_{c s}(1+\mu)},
$$

with $\mu>1$, and $\gamma_{c s}>0$ ensuring the local asymptotic stability of the fixed point of the feedback transformed system when $v=0$.

\section{Conclusions}

The discrete-time version of the SG algorithm has been adapted in order to render passive MIMO nonlinear discretetime systems which are affine in the input. A subclass of passive systems have been introduced, addressed as quasi- $V$-passive systems. Two kinds of feedback passivation schemes have been obtained: a dynamic one and a static one. The feedback passivation methodology proposed is based on the establishment of the input $u$ which locally satisfies the basic passivity equality, which is interpreted as a goal function. Two SISO examples have illustrated the methodology. Some comments on the frequency-domain implications of linear quasi- $V$-passive systems have also been pointed out.

\section{References}

Byrnes, C. I., Isidori, A., \& Willems, J. C. (1991). Passivity, feedback equivalence, and the global stabilization of minimum phase nonlinear systems. IEEE Transactions on Automatic Control, 36, 1228-1240.
Byrnes, C. I., \& Lin, W. (1994). Losslessness, feedback equivalence, and the global stabilization of discrete-time nonlinear systems. IEEE Transactions on Automatic Control, 39(1), 83-98.

Fradkov, A. L. (1991). Speed-gradient laws of control and evolution. In European control conference (pp. 1861-1865).

Fradkov, A. L., \& Pogromsky, A. Y. (1998). Introduction to control oscillations and chaos. Singapore: World Scientific Publishing Co.

Hill, D. J., \& Moylan, P. (1980). Dissipative dynamical systems: Basic input-output and state properties. Journal of the Franklin Institute, 309, 327-357.

Hitz, L., \& Anderson, B. D. O. (1969). Discrete positive-real functions and their application to system stability. Proceedings of IEE, 116, 153-155.

Jiang, Z. P., Lin, Y., \& Wang, Y. (2004). Nonlinear small-gain theorems for discrete-time feedback systems and applications. Automatica, 40, 2129-2136.

Marsden, J. E., \& Hoffman, M. J. (1993). Elementary classical analysis. (2nd ed.), New York: W.H. Freeman Publishers.

Navarro-López, E. M. (2007). QSS-dissipativity and feedback QS-passivity of nonlinear discrete-time systems. Dynamics of Continuous Discrete and Impulsive Systems-Series B: Applications and Algorithms, 14(1), 47-63.

Navarro-López, E. M., \& Fossas-Colet, E. (2004). Feedback passivity of nonlinear discrete-time systems with direct input-output link. Automatica, 40(8), 1423-1428.

Navarro-López, E. M., Sira-Ramírez, H., \& Fossas-Colet, E. (2002). Dissipativity and feedback dissipativity properties of general nonlinear discrete-time systems. European Journal of Control (Special Issue: Dissipativity of Dynamical Systems. Application in Control), 8(3), 265-274.

Nešić, D., \& Grüne, L. (2005). Lyapunov-based continuous-time nonlinear controller redesign for sampled-data implementation. Automatica, 41, $1143-1156$.

Nešić, D., \& Teel, A. R. (2004). A framework for stabilization of nonlinear sampled-data systems based on their approximate discrete-time models. IEEE Transactions on Automatic Control, 49(7), 1103-1122.

Sira-Ramírez, H. (1991). Non-linear discrete variable structure systems in quasi-sliding mode. International Journal of Control, 54(5), 1171-1187.

van der Schaft, A., \& Schumacher, H. (2000). An introduction to hybrid dynamical systems. London: Springer. 